${ }^{3}$ Hirschfelder, J. D., Curtiss, C. F., and Bird, R. B., Molecular Theory of Gases and Liquids, 252 (Wiley, New York, 1954).

4 Flory, P. J., Principles of Polymer Chemistry, ch. 12 (Cornell University Press, New York, 1953).

\section{Load-bearing structures and crystal intergrowth}

As far as I am aware, no one has attempted to place the concept of crystal-crystal intergrowth on a quantitative basis. The object of this note is to calculate the probability of the formation of a three-dimensional load-bearing structure on the basis of crystal-crystal intergrowth.

The origin of the load-bearing capacities of structures like inorganic cements and bone is still disputed. In each of these structures, themselves composites, the solid component, generally assumed to be the main load-bearing element, is mainly crystalline in nature, though usually of submicrometre size. Two mechanisms have been proposed to explain loadbearing capacities. In the first it is assumed that the solid particles form a physically bonded structure ${ }^{1-3}$ whereas the other assumes that the solid particles form a three-dimensional structure by crystal-crystal intergrowth at the points of contact $^{4-7}$. In either case, the points of contact or the points of intergrowth must be numerous and spread throughout the volume for the structure to be functional.

In inorganic cements as well as in bone, crystallisation begins in isolated regions. Subsequent crystallisation and crystal growth bring the crystals from different regions into contact. Thus, when two crystals from two different regions meet, they do so at a random orientation. The case of apatite crystals in long bones is somewhat complicated. The apatite crystals in long bones of a newly born baby are randomly oriented; those in mature bone, however, have a preferred orientation with the $c$ axis parallel to the long axis of the bone ${ }^{8}$.

When two crystals from two different regions come into contact two possibilities may arise. In the furst case, the atoms at the interface can be considered to belong to the crystal structures of both crystals and because of the structural continuity the intercrystalline bond is nearly as strong as the intracrystalline bond. In the second case there is no such relationship and the intercrystalline bond is much weaker than the intracrystalline bond so the crytsals can be considered to be physically bonded.

For ideal crystals, crystal-crystal intergrowth can only occur when the crystals meet each other in a symmetry related indistinguishable orientation. For micrometre sized real crystals some angular relaxation of the order of the mosaic spread in a single crystal-that is $3-4^{\circ}-$ is expected. The probability that two crystals will neet each other in this orientation, by accident, can be calculated.

Let (hkl) be the crystal plane across which the crystal-crystal intergrowth is going to occur. Let $c$ be an axis normal to the plane having an $n$ degree rotation symmetry and $a$ be an axis in the plane. If a second crystal is brought into contact with the first an intergrowth will occur when both $c$ and $a$ axes of the two crystals are within the angular relaxation zones of each other. Statistically, the second $c$ axis can approach the first from any point on a hemisphere drawn round the first. The probability of two $c$ axes being within the relaxation cones of each other is given by

$$
P_{c}=\left(1-\cos 4^{\circ}\right)=0.002
$$

The $a$ axis will repeat $n$ times in $360^{\circ}$, so that when two $c$ axes are parallel to each other the probability of the two $a$ axes coming within each other's relaxation zones is given by

$$
P_{a}=n \times 4^{\circ} / 360^{\circ}=n / 90
$$

so that the total probability of intergrowth is

$$
P_{t}=P_{c} P_{\mathrm{a}}=n / 45,000
$$

The probability of a third crystal joining either of the first two in intergrowth orientation is given by $\boldsymbol{P}_{\mathrm{t}}{ }^{2}$ and so on. Thus the probability of the formation of an extended structure on this basis is vanishingly small. Even if most of the $c$ axes are parallel to each other, somewhat like apatite crystals in mature bone, the probability that a load bearing structure will form will still be extremely small. The probability of obtaining an aggregate $1 \mathrm{~mm}$ long from micrometre-sized crystals on this basis will be $(n / 90)^{999}$ where $n$ is a small integer. This very low probability is consistent with a scanning electron microscopic work on plaster of Paris blocks ${ }^{9}$. This work did not reveal any definite evidence for the occurrence of extensive crystal-crystal intergrowth though one of the objects of the study was to search for it.

In the case of physical bonding of the crystals there is no requirement of any continuity of atomic arrangement between the participating crystals, so bonding will occur whenever crystals come sufficiently close to each other. The probability of the formation of an extensive structure on this basis depends only on the packing density of the crystals and on their size and shape $^{10}$.

Thus the most probable mechanism of the origin of loadbearing capacities of bone and inorganic cements is the physical bonding of the crystals.

I thank Professor A. Tovborg Jensen, Landbohojskolen, and Director $\mathrm{P}$. Nernest, $\mathrm{H}+\mathrm{H}$ Industri $\mathrm{A} / \mathrm{S}$, for their interest in this work.

\section{S. Chatterji}

Department of Inorganic Chemistry, Landbohojskolen, Copenhagen, Denmark

Received April 25; revised July 25, 1974.

Permanent address: $\mathrm{H}+\mathrm{H}$ Industri A/S, 2800 Lyngby, Denmark.

${ }^{1}$ le Chatelier, H., Experimental Researches on the Constitution of Hydraulic Mortars, Translated by J. L. Mack (McGraw-Hill, New York, 1905).

${ }^{2}$ Chatterji, S., and Jeffery, J. W., Nature, 214, 556 (1967).

${ }^{3}$ Chatterji, S. and Jeffery, J. W., Nature, 219, 482 (1968).

${ }^{4}$ Currey, J. D., J. Biomech., 2, 477 (1969).

${ }^{5}$ Feldmann, R. F., and Sereda, P. J., Proc. 5 th int. Symp. Chemistry of Cement, Tokyo, 3, (3), 36 (1968).

${ }^{6}$ Matkovic, B., and Young, J. F., Nature phys. Sci., 246, 79 (1973).

${ }^{7}$ Piekarski, K., Int. J. eng. Sci., 11, 557 (1973).

${ }^{8}$ Chatteri, S., et al., Experientia, 28, 156 (1972).

${ }^{9}$ Chatterji, S., et al., Cem. Technol., 1, 171 (1970).

${ }^{10}$ Mamedov, H. S., et al., Indian Concr. J., 42, 66 (1968).

\section{Direct observation of alkali metal colloids in alkali halide crystals}

SiEDENTOPF ${ }^{1}$ observed that sodium chloride crystals containing atomically dispersed $F$ centres turned blue and exhibited Tyndall scattering when annealed at $400^{\circ} \mathrm{C}$. These effects were attributed to colloidal particles of sodium metal formed in $\mathrm{NaCl}$ crystals by aggregation of $\mathrm{F}$ centres during the annealing process. Savostianova ${ }^{2}$ applied the $\mathrm{Mie}^{3}$ theory to absorption and scattering of light from colloidal metallic particles suspended in dielectric media and predicted colloid sizes in excess of $80 \mathrm{~nm}$. The blue colour of natural rocksalt, investigated $^{4}$ using optical absorption and photoconductivity, was similarly attributed to colloids. Ionising radiation also produces colloidal absorption bands in irradiated alkali halide crystals under certain conditions $\mathrm{s}^{5-10}$.

Increasingly sophisticated techniques have been applied to the study of these colloidal centres, for example, ultramicro- 